\title{
Modelo Multicriterio para el Diseño de Cadenas de Suministro Considerando Opciones Reales para el Tratamiento de la Incertidumbre
}

\author{
Multi-Criteria Model for the Design of Supply Chains Considering Real Options \\ for the Treatment of Uncertainty
}

\author{
V. J. Jiménez-Carabalí iD ; D. F. Manotas-Duque iD , L. Rivera-Cadavid iD \\ DOI: https://doi.org/10.22517/23447214.24543 \\ Artículo de investigación científica y tecnológica
}

\begin{abstract}
Resumen - Los problemas relacionados con la configuración de cadenas de suministros incluyen variables cuantitativas y cualitativas. Entre los atributos cuantitativos el más común es el dinero, y muchos atributos como la utilización de las máquinas, la fuerza de trabajo y la calidad pueden traducirse en términos monetarios. Algunos ejemplos de variables cualitativas son el estado de ánimo de las personas, la satisfacción del cliente, la reputación en la comunidad, la apariencia, la estética, etc. Los modelos de decisión de atributos múltiples integran los atributos cuantitativos y cualitativos para producir una medida de desempeño agregada En este artículo se propone un modelo de decisión multicriterio utilizando basado en el Analytical Hierarchy Procces (AHP) para la configuración de cadenas de abastecimiento integrando elementos de flexibilidad financiera a través del análisis de opciones reales. El modelo desarrollado contempla elementos de riesgo financiero a partir de la consideración de variables como demanda, tasas de cambio y tasas de interés, y permite valorar la flexibilidad operativa del proyecto ante cambios en dichos elementos de riesgo.
\end{abstract}

Palabras Claves - AHP, Flexibilidad, Gestión de Cadenas de Suministro, Opciones Reales.

Abstract-The problems related to the configuration of supply chains include quantitative and qualitative variables. Among the quantitative attributes, the most common is the money, and many attributes such as machine utilization, labor power and quality can be translated into monetary terms. Some examples of qualitative variables are people's mood, customer satisfaction, reputation in the community, appearance, aesthetics, etc. Multiple-attribute decision models integrate quantitative and qualitative attributes to produce an aggregate performance measure. This paper proposes a multi-criteria decision model using the Analytical Hierarchy

Este manuscrito fue enviado el 27 de octubre de 2020 y aceptado 23 de junio 2021.

V. J. Jiménez-Carabalí. Profesor Asistente. Departamento de Contabilidad y Finanzas, Universidad del Valle. Doctorado en Engenharia Industrial e Sistemas, Universidade do Minho. Magister en Ingeniería e Ingeniero Industrial de la Universidad del Valle. (e-mail: victor.jimenez@correounivalle.edu.co).

D. F. Manotas-Duque. Profesor Titular. Escuela de Ingeniería Industrial, Universidad del Valle. Doctor en Ingeniería, Universidad del Valle. Magíster
Process (AHP) for the configuration of supply chains by integrating elements of financial flexibility through the analysis of real options. The model developed considers elements of financial risk based on the consideration of variables such as demand, exchange rates and interest rates, and allows assessing the operational flexibility of the project in the face of changes in said risk elements.

Index Terms - AHP, Flexibility, Supply Chain Management, Real Options.

\section{INTRODUCCIÓN}

$\mathrm{L}$ A gestión financiera de las cadenas de suministros es un tópico de investigación relativamente reciente. El propósito fundamental es la optimización de flujos financieros a nivel interorganizacional [1], alineando los flujos financieros con los flujos de productos e información dentro de la cadena, procurando mejorar la gestión del flujo de efectivo a lo largo de los diferentes eslabones.

Las cadenas de suministro además de satisfacer al cliente tienen como objetivo maximizar el valor total generado. El valor medido como la diferencia entre lo que vale el producto final para el cliente y los costos en que la cadena incurre para cumplir la petición de éste, esto es la utilidad que se repartirá entre todas las etapas e intermediarios de la cadena. Para

en Gestión de Empresas, Universidad de Chile. Ingeniero Industrial y Especialista en Finanzas, Universidad del Valle. (email: diego.manotas@correounivalle.edu.co).

L. Rivera-Cadavid. Profesor Asistente. Escuela de Ingeniería Industrial, Universidad del Valle. Ph.D. in Industrial and Systems Engineering, Virginia Polytechnic Institute and State University. M.Sc. Industrial and Systems Engineering, Georgia Institute of Technology. Ingeniero Industrial Universidad del Valle. (e-mail: leonardo.rivera.c@ correounivalle.edu.co). 
cualquier cadena de suministros existe una sola fuente de ingresos, el cliente, todos los demás flujos de efectivo son simplemente intercambio de fondos que ocurren dentro de la cadena, dado que las diferentes etapas tienen diferentes dueños. Por lo tanto, la administración adecuada de estos flujos es una de las claves del éxito de la cadena de suministro [2].

Las decisiones en el diseño de la cadena de suministros tales como el número y el tamaño de las plantas a construir, el tamaño y el alcance del sistema de distribución y si comprar y/o arrendar las instalaciones a utilizar suponen inversiones significativas. Estas decisiones, una vez que se toman, no pueden alterarse en el corto plazo, permanecen en vigor varios años y definen las restricciones dentro de las cuales la cadena deberá competir. Por tanto, es importante que sean evaluadas con suma precisión [2].

Otros criterios importantes que se deben tener en cuenta son criterios de carácter cualitativo, entre esos se encuentran el posicionamiento de marca, el nivel de servicio, las políticas gubernamentales, la infraestructura pública, recursos humanos, financieros y de información, entre otros [3], [4]. Criterios difíciles de medir mediante modelos matemáticos convencionales [5], [6]; y que además generan riesgo e incertidumbre a la toma de decisiones para cada uno de los eslabones de las cadenas de suministro. Por otra parte la gestión del riesgo en cadenas de suministro es un campo creciente, que busca desarrollar un enfoque para identificar, evaluar y tratar las áreas de vulnerabilidad y riesgo presente en las cadenas [7][9]. Muchas de las metodologías para la gestión de los riesgos se enfocan en la predicción de las rupturas en las cadenas en vez de enfocarse en la raíz de las causas de la incertidumbre[10].

En la configuración clásica de cadenas de suministro, uno de los objetivos primordiales tiene que ver con la minimización de los costos o maximización de las utilidades, esto bajo el supuesto que existe certidumbre y que las decisiones tomadas en cualquier momento son difíciles de modificar sino hasta largos periodos de tiempo, lo cual no es necesariamente cierto, debido a la existencia de incertidumbre en las variables exógenas, tales como: la inflación, las variaciones en la demanda, en los precios y en las tasas de mercado [10]-[12].

Es por esto que además de los requisitos esenciales para el diseño de cadenas de suministro, se deben incluir componentes relacionados con la flexibilidad asociada a los ambientes de incertidumbre en los cuales se desenvuelven las organizaciones actualmente, teniendo en cuenta que la flexibilidad conlleva costos, que deben ser valorados y contrastados con los beneficios que se puedan generar. [1], [13], [14] .

Respecto a la flexibilidad [15]-[17] mencionan que a menudo las inversiones siguen una secuencia natural de pasos con múltiples puntos de decisión con respecto a continuar, postergar o abandonar, permitiendo a una organización responder a cambios en el mercado o a las reglas del gobierno o adaptarse a avances tecnológicos, es decir siempre existen opciones alternativas de decisión. La no consideración de las opciones implícitas en un proyecto conlleva a subvalorarlo y en general a desechar proyectos que se deberían realizar dado las opciones futuras relacionadas con el desempeño del entorno. De forma análoga si un proyecto implica opciones que podrían ejercer terceros, la flexibilidad futura del mismo podría tener impactos negativos sobre el desempeño financiero del proyecto [18]. Todos estos elementos hacen que la gestión de las cadenas de suministro sea una tarea compleja y cambiante debido a la expansión en la variedad de productos, la disminución en el ciclo de vida de los productos, el incremento del outsourcing y los continuos avances en la tecnología de la información [19], por lo que el reto del proceso de gestión es el desarrollo de metodologías para la toma de decisiones que se adapten a los diferentes agentes de la cadena de suministro [5] .Todas estas consideraciones introducen una gran cantidad de variables y restricciones, agregando complejidad a la toma de decisiones, complejidad que no puede ser abordada simplemente desde la intuición o la experiencia. Es por esto que se deben buscar herramientas que permitan establecer configuraciones adecuadas, en periodos de tiempo considerablemente cortos, que se adecúen a las exigencias tanto de los clientes, como del mercado en el cual se desarrollan. En este artículo mostramos como se pueden incorporar diferentes criterios cuantitativos y cualitativos a la hora de configurar una cadena de suministros utilizando la metodología multicriterio Analytical Hierarchy Procces (AHP) [20]-[22] y destacando principalmente la flexibilidad financiera a través de la evaluación de opciones reales. Encontramos que la flexibilidad que se tenga para cambiar a la configuración de una cadena de suministros en un periodo determinado aumenta el valor de este tipo de proyectos.

\section{METODOLOGÍA PROPUESTA}

Para la construcción de la metodología se debe tener en cuenta que las decisiones dependen del horizonte de planeación, por ejemplo para la fase estratégica se deben elegir todos los posible lugares donde se podrían ubicar cada uno de los eslabones principales de la cadena, incluyendo las zonas de consumo, luego de esto se procede a utilizar un modelo de localización con el fin de proveer la distribución física de la red, teniendo en cuenta los criterios relevantes para la toma de decisiones a lo largo de la cadena de suministro, con el fin de lograr la satisfacción del cliente.

Una vez definida la estrategia se procede a la fase tácticooperativa, allí, la organización debe tratar de evaluar las variables que generadoras de incertidumbre tales como la demanda, la oferta, las tasas de cambio, la capacidad, etc., y ver en qué medida se puede aprovechar al máximo dichas variables. Una vez son identificadas las variables se deben encontrar todas aquellas opciones reales que la empresa posee, y que puede utilizarse para el mejoramiento de sus medidas de desempeño, se podría decir que, en cadenas de suministro, dichas opciones se presentan más a nivel táctico-operativo, dada la facilidad que se tienen para afectar el sistema con decisiones en el corto plazo. Luego de identificar dichas opciones se deben valorar, y, los resultados obtenidos se deben contrastar frente a los 
objetivos estratégicos de la cadena de suministro. La figura 1 ilustra gráficamente los pasos para la metodología propuesta.

\begin{tabular}{|c|}
\hline $\begin{array}{l}\text { 1. Identificación de zonas para la ubicación } \\
\text { de los eslabones de la cadena de suministro. }\end{array}$ \\
\hline$\nabla$ \\
\hline 2. Modelo de localización. \\
\hline$\nabla$ \\
\hline $\begin{array}{l}\text { 3. Identificación de criterios de decisión } \\
\text { relevantes. }\end{array}$ \\
\hline$\nabla$ \\
\hline $\begin{array}{l}\text { 4. Identificación de las opciones reales para } \\
\text { las potenciales configuraciones existentes. }\end{array}$ \\
\hline$\checkmark$ \\
\hline $\begin{array}{l}\text { 5. Valoración de las opciones reales } \\
\text { identificadas. }\end{array}$ \\
\hline$\checkmark$ \\
\hline $\begin{array}{l}\text { 6. Análisis de sensibilidad de las opciones } \\
\text { reales, teniendo en cuenta las variables que } \\
\text { influyen en su valor. }\end{array}$ \\
\hline$\checkmark$ \\
\hline $\begin{array}{l}\text { 7. Modelo de simulación de Monte Carlo } \\
\text { para análisis de riesgo. }\end{array}$ \\
\hline$\nabla$ \\
\hline $\begin{array}{l}\text { 8. Modelo multicriterio (AHP) para la } \\
\text { selección de configuraciones de la cadena de } \\
\text { suministro. }\end{array}$ \\
\hline
\end{tabular}

Fig. 1. Pasos de la metodología propuesta

Dicha metodología consta de 8 pasos los cuales se describen a continuación:

Identificar las posibles zonas donde se ubicarían los potenciales eslabones de la cadena de suministro: En esta etapa se espera que la organización evalúe teniendo en todos los puntos posibles en donde puede localizar cada uno de los diferentes componentes de la cadena de suministros, para dicha evaluación se deben tener en cuenta factores como los costos de estar en cierta localización, el modo de transporte requerido, aspectos tributarios, entre otros.

Formular un modelo de localización con el fin de establecer la ubicación física. Una vez identificados todos los puntos, se debe formular un modelo que permita establecer la ubicación de cada uno de los eslabones de la cadena, principalmente la ubicación de plantas, centros de distribución y zonas de consumo.

Identificar los criterios relevantes para la toma de decisiones sobre las necesidades del cliente. Se deben establecer criterios relacionados con la estrategia de la organización en lo relacionado con las necesidades del cliente, en esta etapa se deben definir aspectos tales como: si la cadena estará orientada en la eficiencia, en la capacidad de respuesta, en la calidad, en la maximización de la utilidad global, o de cada uno de los eslabones.
Identificar las opciones reales para las potenciales configuraciones existentes: Una vez establecidas las configuraciones viables, el paso siguiente es identificar todos aquellos puntos donde la organización pueda cambiar en un determinado momento el rumbo de sus operaciones, afectando así su desempeño futuro, dicha flexibilidad puede estar indicando la existencia de una "opción real", por ejemplo si la organización en un determinado momento puede no operar cuando los resultados sean adversos, se está ante una opción de abandono, por el contrario si dadas unas condiciones favorables las empresa tiene un mercado cautivo y puede ampliar su capacidad en un determinado momento para satisfacer las necesidades de dicho mercado, se está frente a una opción de expansión, cabe destacar que la identificación de las opciones es un proceso complejo que requiere un amplio conocimiento de los movimientos de una organización.

Valorar las opciones: Una vez identificadas las opciones, se procede a realizar la valoración "determinística" de cada una de las configuraciones con y sin opciones, para esto se puede utilizar un modelo de árboles binomiales o multinomiales, o el modelo de Black-Scholes, se destaca que cuando las opciones poseen múltiples subyacentes, la elaboración de los árboles puede ser muy difícil y un proceso casi impráctico, por lo que en ese caso se recomienda la utilización del modelo generalizado de B-S o del modelo de Samuelson propuesto por [23] el cual reduce el problema de múltiples variables a un problema que se puede modelar utilizando un árbol binomial.

Realizar un análisis de sensibilidad a las opciones valoradas: Al valorar las opciones se deben identificar aquellas variables que puedan impactar en el desempeño futuro de las configuraciones potenciales, es decir las variables que generan incertidumbre en el proceso de decisión. Entre estas variables se encuentran la demanda de los centros de consumo, la oferta de los proveedores, las tasas de cambio, los tiempos de espera, entre otras; después de ser identificadas, aquellas que son relevantes para cada configuración, deben ser caracterizadas desde el punto de vista estadístico, esto es definir una distribución de probabilidad asociada, la cual puede ser la distribución empírica de una serie de datos o determinada a priori dado el conocimiento específico que se tienen de los procesos realizados en la cadena de suministro.

Formular un modelo de simulación: Una vez identificadas las variables que generan incertidumbre, se procede a realizar un modelo de simulación con el cual se pueda evaluar los posibles resultados para cada una de las configuraciones existentes, esto con el fin de determinar estadísticas de tendencia central y de dispersión principalmente, sobre ciertas medidas de desempeño las cuales están relacionadas con los costos, o con las utilidades.

Construir un modelo AHP como elemento para la toma de decisiones: Una vez corrido el modelo de simulación, se procede a construir un modelo AHP con cada una de las posibles configuraciones existentes, para ello se deben tener en cuenta los criterios definidos en la etapa 3 y los resultados obtenidos en la etapa 7, se espera que en esta etapa se pueda tomar una decisión acorde a los objetivos establecidos por la o las organizaciones implícitas en la cadena de suministro. El AHP permite a los decisores visualizar la estructura de un 
problema complejo en forma de una jerarquía tendiendo como mínimo dos niveles; objetivos (criterios para evaluación) y actividades (productos y alternativas) para posteriormente cuantificar prioridades relativas del conjunto de alternativas sobre una escala de razón. De aquí que se concentre en la

importancia de los juicios intuitivos, así como en la consistencia de la comparación de las alternativas en el proceso de decisión La fuerza de este método se debe, entre otras razones, a que: organiza factores tangibles e intangibles de un modo sistemático proporcionando una solución simple pero estructurada, y además cada factor o alternativa sobre un nivel dado puede ser identificado y evaluado con respecto a otros factores relacionados. Así pues, el AHP estructura un problema y enfoca la atención en componentes específicos de tal manera que se amplían las capacidades de toma de decisión[9], [21] .

\section{CASO DE ESTUdiO}

Después de analizar el mercado y las posibilidades de localización de sus plantas, una empresa con casa matriz en los Estados Unidos - donde consolida sus utilidades- y dedicada a la comercialización de un producto masivo ha decidido que se concentrará en el mercado de Estados Unidos y Colombia. Por políticas de la compañía se úbicará un centro producción en cada uno de los países, y actualmente está interesada en saber cómo debe configurar su cadena de suministro de tal manera que maximice el valor económico y además esté alineada con la estrategia y objetivos de la organización. Dicha decisión debe tomarse teniendo en cuenta que las condiciones del mercado presentan tasas de cambio fluctuantes e incertidumbre en la demanda.

La demanda en Colombia cada año se comporta de acuerdo con una distribución normal que tiene media 500.000 y desviación estándar 100.000 y en los Estados Unidos con una distribución normal con media 800.000, y desviación estándar 100.000. Las fluctuaciones de la demanda para ambos países son independientes.

Una unidad de producto se vende en 30 dólares en Estados Unidos y 108.000 pesos en Colombia, dicho precio se mantiene constante al pasar los años. La tasa de cambio actual es de 3.600 pesos por dólar, se espera que la tasa de cambio fluctué de acuerdo con la distribución normal con media 3600 y desviación estándar 50. Las fluctuaciones en la tasa de cambio son independientes de las fluctuaciones en la demanda.

La organización sabe que tendrá una planta para cumplir con las especificaciones de la demanda, en cada país. Cada una de las plantas puede ser dedicada o flexible, en el primer caso, solo podrá atender el mercado local; en el segundo caso, podrán abastecer cualquiera de los dos mercados, esto básicamente se presenta porque las especificaciones de producto son diferentes en cada país, y a pesar de que es el mismo producto se deben realizar ajustes en el empaque que cumplan con los requisitos establecidos en el país al cual se realiza la exportación. Los costos fijos y variables de cada opción se muestran en la tabla I.
TABLA I

PARÁMETROS INICIALES DEL CASO

\begin{tabular}{|c|c|c|c|c|c|c|c|}
\hline & $\begin{array}{c}\text { Capa } \\
\text { cidad } \\
\text { [Uni./ } \\
\text { año] }\end{array}$ & $\begin{array}{c}\text { Costo de } \\
\text { envío } \\
\text { desde } \\
\text { planta } \\
\text { [USD] }\end{array}$ & $\begin{array}{c}\text { Planta } \\
\text { dedicada }\end{array}$ & $\begin{array}{c}\text { Planta } \\
\text { flexible }\end{array}$ & & & \\
\hline Planta & & & $\begin{array}{l}\text { Costo } \\
\text { fijo }\end{array}$ & $\begin{array}{l}\text { Costo } \\
\text { fijo }\end{array}$ & $\begin{array}{l}\text { Co } \\
\text { sto } \\
\text { var } \\
\text { iab } \\
\text { le }\end{array}$ & $\begin{array}{l}\text { Preci } \\
\text { o de } \\
\text { venta }\end{array}$ & $\begin{array}{l}\text { Mone } \\
\text { da }\end{array}$ \\
\hline $\begin{array}{l}\text { Estados } \\
\text { Unidos }\end{array}$ & $\begin{array}{l}1.000 \\
.000\end{array}$ & 3 & $\begin{array}{l}1.000 .00 \\
0\end{array}$ & $\begin{array}{l}1.100 .00 \\
0\end{array}$ & 15 & 30 & USD \\
\hline $\begin{array}{l}\text { Colombi } \\
\mathrm{a}\end{array}$ & $\begin{array}{l}400.0 \\
00\end{array}$ & 3 & $\begin{array}{l}1.800 .00 \\
0.000\end{array}$ & $\begin{array}{l}1.980 .00 \\
0.000\end{array}$ & $\begin{array}{l}54 . \\
00 \\
0\end{array}$ & $\begin{array}{l}108.0 \\
00\end{array}$ & $\begin{array}{l}\text { PES } \\
\text { OS }\end{array}$ \\
\hline
\end{tabular}

Los costos fijos están dados por año. Los costos de transporte entre Estados Unidos y Colombia están dados en un dólar por cada unidad indiferente del país de origen. La tasa de descuento empleada es de 5\% anual.

Adicionalmente la organización puede tomar la decisión de cambiar sus plantas de dedicadas a flexibles y viceversa de un periodo a otro, incurriendo en unos costos de cambio tal como se muestran en la tabla II, todas las unidades están en dólares.

TABLA II

COSTO DE CAMBIO DE LAS COMBINACIONES POSIBLES

\begin{tabular}{lcccc}
\hline \hline & UD-CD & UF-CF & UF-CD & UD-CF \\
\hline UD-CD & 0 & 15.750 & 10.500 & 5.250 \\
UF-CF & 15.750 & 0 & 5.250 & 10.500 \\
UF-CD & $10-500$ & 5.250 & 0 & 15.750 \\
UD-CF & 5.250 & 10.500 & 15.750 & 0 \\
\hline \hline
\end{tabular}

En la tabla II U hace alusión a Estados Unidos, C a Colombia, $\mathrm{D}$ a dedicada y $\mathrm{F}$ a flexible así por ejemplo UD-CD significa que las dos plantas tanto de Estados Unidos como de Colombia son dedicadas.

Dichos costos fueron calculados asumiendo que el costo de cambio se relaciona con el costo fijo de operar una planta, así para pasar de dedicada a flexible, el costo está alrededor del $1 \%$ y 5\% del costo promedio de operación - se asume una distribución uniforme- y del flexible a dedicada entre el $1 \%$ y el 5\% también con una distribución uniforme.

En este caso se identifican dos tipos de opciones reales, la primera tiene que ver con la opción de abandono de la operación, la cual se presenta en caso de que los costos superen los ingresos en un determinado periodo, en cuyo caso la empresa optaría por no atender sus clientes. La segunda tiene que ver con una opción de cambio entre un periodo y otro, dadas algunas condiciones específicas de la demanda, la organización puede optar por transformar sus plantas de flexibles a dedicadas o viceversa, claro está incurriendo en los costos mostrados en la tabla II.

Debido a que se cuenta con tres variables se puede construir un árbol multinomial donde cada nodo en un periodo dado lleva a ocho nodos posible en el siguiente, ya que la demanda en cada país y la tasa de cambio pueden disminuir o aumentar en cada periodo. 
El problema que se evidencia al manejarlo así tiene que ver con la complejidad primero en la cantidad de ramas que se derivan de un nodo, eso es $2^{n}$ donde $\mathrm{n}$ es el número de variables que para este caso es 3 , es decir en un problema con solo 20 variables ya se puede considerar inmanejable desde el punto de vista práctico. Otro problema que se evidencia tiene que ver con el cálculo de las probabilidades de las ramas y de lo cual todavía es objeto de estudio cuando se desea manejar las incertidumbres de manera independiente. - esta representación es pensada en árboles multinomiales probabilidades independientes entre las variables.

Otra estrategia de solución tiene que ver con la consolidación de las variables originadoras de riesgo en una función de utilidad, y el cálculo de la volatilidad sobre dicha función, lo cual recoge la volatilidad de todas las variables, y reduce el problema a un árbol binomial, en el cual se puede aplicar la teoría de opciones reales tradicional.

La opción de cambio tiene en cuenta todas las posibles combinaciones, es decir ambas flexibles, ambas dedicadas, Colombia flexible y Estados Unidos dedicada, o Colombia dedicada y Estados Unidos flexible en incluye los costos de cambio de una alternativa a otra.

Una vez conocidos las posibles decisiones que se pueden tomar, el paso siguiente es calcular la mayor utilidad considerando la opción de abandono para cada uno de los periodos establecidos en el horizonte de planeación, que en este caso se asumen 5 años, dado que es una decisión estratégica. Para dicho proceso se debe tener en cuenta los siguientes parámetros.

Para la opción de cambio en el periodo cero se debe establecer un nodo de arranque, a partir del cual se empiezan a tomar las decisiones futuras.

Así por ejemplo cuando se inicia con la configuración Estados Unidos dedicada y Colombia dedicada la utilidad de cada uno de los periodos es equivalente a la máxima utilidad obtenida en la configuración Dedicada- Dedicada, con las condiciones particulares de dicho periodo.

Una vez obtenidas las utilidades por periodo, se procede a calcular el valor presente de cada una de las utilidades para cada configuración tanto en el periodo cero, como el periodo uno, con el objetivo de calcular la variación del proyecto como sigue:

$$
\text { variación logarítimica de la configuración }=L N \frac{V P U_{1}}{V P U_{O}}
$$

Donde VPU $\mathrm{V}_{1}$ Es igual al valor presente de la utilidad en el periodo 1 y Donde $\mathrm{VPU}_{0}$ es el valor presente de la utilidad en el periodo cero.

Entonces la volatilidad de cada configuración estará dada por la desviación estándar de dichas variaciones logarítmicas, dicha desviación será un resultado obtenido después de un proceso de simulación.

Una vez obtenida la desviación estándar, se procede a calcular los parámetros para la elaboración de un árbol binomial. Los parámetros principales son los coeficientes de ascenso y descenso y las probabilidades asociadas a ellas.

Para el cálculo del valor de la opción de cambio se deben tener en cuenta las siguientes variables:

$Z_{U_{f}^{d}-C_{f}^{d}}^{n_{b}^{a}}$ La variable indica el valor presente de la utilidad al alza o a la baja en el periodo $n$, donde $n=0,1,2$. Dada por cualquiera de las cuatro combinaciones posibles de plantas flexibles y dedicadas.

$r_{u_{d}^{u}}^{c_{d}^{u}}$ : Indica el costo de cambio de una planta flexible a una planta dedicada, de una planta dedicada a una dedicada, de una planta flexible a una flexible, y de una planta flexible a una planta dedicada.

$W_{n}$ : Valor presente de la utilidad en el periodo $\mathrm{n}$.

$v_{U_{d}^{u}-C_{f}^{d}}$ : Valor presente de la utilidad en el periodo cero.

$U_{U_{f}^{d}-C_{f}^{d}}$ : Indica el coeficiente de ascenso de cada una de las configuraciones posibles

$D_{U_{f}^{d}-C_{f}^{d}}$ : Indica el coeficiente de descenso de cada una de las configuraciones posibles.

Con estas variables se calcula la utilidad en cada uno de los nodos del árbol binomial teniendo en cuenta el periodo, para el nodo del periodo cero asumiendo que se inicia con las dos plantas dedicadas se tiene que:

Para los del perido1:

$$
W_{0}=v_{u d-c d}
$$

El valor de la utilidad al alza esta dado por:

$$
\begin{gathered}
W_{1}=M a ́ x\left(W_{0} * U_{u d-c d}-r_{u d-u d}^{c d-c d} ; Z_{u f-c f}^{1^{a}}-r_{u d-u f}^{c d-c f} ; Z_{u f-c d}^{1^{a}}\right. \\
\left.-r_{u d-u f}^{c d-c d} ; Z_{u d-c f}^{1^{a}}-r_{u d-u d}^{c d-c f}\right)
\end{gathered}
$$

El valor de la utilidad a la baja esta dado por:

$$
\begin{gathered}
W_{1}=M a ́ x\left(W_{0} * D_{u d-c d}-r_{u d-u d}^{c d-c d} ; Z_{u f-c f}^{1^{b}}-r_{u d-u f}^{c d-c f} ; Z_{u f-c d}^{1^{b}}\right. \\
\left.-r_{u d-u f}^{c d-c d} ; Z_{u d-c f}^{1^{b}}-r_{u d-u d}^{c d-c f}\right)
\end{gathered}
$$

Partiendo de la configuración de dos plantas flexibles de tiene que:

Para el periodo 0.

$W_{0}=v_{u f-c f}$ (5)

Para el periodo 1.

El valor de la utilidad al alza esta dado por:

$$
\begin{gathered}
W_{1}=M a ́ x\left(Z_{u d-c d}^{1^{a}}-r_{u f-u d}^{c f-c d} ; W_{0} * U_{u f-c f}-r_{u f-u f}^{c f-c f} ; Z_{u f-c d}^{1^{a}}\right. \\
\left.-r_{u f-u f}^{c f-c d} ; Z_{u d-c f}^{1^{a}}-r_{u f-u d}^{c f-c f}\right)
\end{gathered}
$$

El valor de la utilidad a la baja esta dado por:

$$
\begin{gathered}
W_{1}=M a ́ x\left(Z_{u d-c d}^{1^{b}}-r_{u f-u d}^{c f-c d} ; W_{0} * D_{u f-c f}-r_{u f-u f}^{c f-c f} ; Z_{u f-c d}^{1^{b}}\right. \\
\left.-r_{u f-u f}^{c f-c d} ; Z_{u d-c f}^{1^{b}}-r_{u f-u d}^{c f-c f}\right)
\end{gathered}
$$


Partiendo de la planta en Estado Unido flexible y en Colombia dedicada se tiene que:

Para el periodo 0.

$$
W_{0}=v_{u f-c d}
$$

Para el periodo 1

$$
\begin{gathered}
W_{1}=M a ́ x\left(Z_{u d-c d}^{1^{a}}-r_{u f-u d}^{c d-c d} ; Z_{u f-c f}^{1^{a}}-r_{u f-u f}^{c d-c f} ; W_{0} * U_{u f-c d}\right. \\
\left.-r_{u f-u f}^{c d-c d} ; Z_{u d-c f}^{1^{a}}-r_{u f-u d}^{c d-c f}\right)
\end{gathered}
$$

El valor de la utilidad a la baja esta dado por:

$$
\begin{gathered}
W_{1}=M a ́ x\left(Z_{u d-c d}^{1^{b}}-r_{u f-u d}^{c d-c d} ; Z_{u f-c f}^{1^{b}}-r_{u f-u f}^{c d-c f} ; W_{0} * D_{u f-c d}\right. \\
\left.-r_{u f-u f}^{c d-c d} ; Z_{u d-c f}^{1^{b}}-r_{u f-u d}^{c d-c f}\right)
\end{gathered}
$$

Partiendo de la planta en Estado Unido dedicada y en Colombia flexible se tiene que:

Para el periodo 0 .

$$
W_{0}=v_{u d-c f}
$$

Para el periodo 1.

El valor de la utilidad al alza esta dado por:

$$
\begin{gathered}
W_{1}=M a ́ x\left(Z_{u d-c d}^{1^{a}}-r_{u d-u d}^{c f-c d} ; Z_{u f-c f}^{1^{a}}-r_{u d-u f}^{c f-c f} ; Z_{u f-c d}^{1^{a}}\right. \\
\left.-r_{u d-u f}^{c f-c d} ; W_{0} * U_{u d-c f}-r_{u d-u d}^{c f-c f}\right)
\end{gathered}
$$

El valor de la utilidad a la baja esta dado por:

$$
\begin{gathered}
W_{1}=M a ́ x\left(Z_{u d-c d}^{1^{b}}-r_{u d-u d}^{c f-c d} ; Z_{u f-c f}^{1^{b}}-r_{u d-u f}^{c f-c f} ; Z_{u f-c d}^{1^{b}}\right. \\
\left.-r_{u d-u f}^{c f-c d} ; W_{0} * D_{u d-c f}-r_{u d-u d}^{c f-c f}\right)
\end{gathered}
$$

Cada una de estas utilidades se pondera por las probabilidades de cada uno de los nodos, tanto los que se encuentran al alza como los que se encuentran a la baja y se descuentan para calcular el valor presente de las utilidades con opción de cambio.

\section{RESULTADOS}

Con toda esta información, se procede a utilizar un software que permita formular un modelo con cada una de las configuraciones existentes y valorar las opciones implícitas en cada una de ellas. Se utilizó Microsoft Excel®. y Risk Simulator ${ }^{\circledR}$. En primera instancia se realiza un análisis determinístico con cada una de las configuraciones posibles cuyo resultado se muestra en la tabla III.

TABLA III

VPN DE LAS UTILIDADES PARA CADA CONFIGURACIÓN CON Y SIN OPCIÓN DE ABANDONO

\begin{tabular}{cccc}
\hline \hline Estados Unidos & Colombia & $\begin{array}{c}\text { Valor presente } \\
\text { de las utilidades }\end{array}$ & $\begin{array}{c}\text { Diferencia } \\
\text { porcentual con } \\
\text { respecto al } \\
\text { máximo }\end{array}$ \\
\hline Dedicada & Dedicada & 71.463 .365 & $6,67 \%$ \\
Flexible & Dedicada & 76.198 .789 & $0,00 \%$ \\
Dedicada & Flexible & 71.219 .891 & $6,99 \%$ \\
Flexible & Flexible & 75.982 .316 & $0,28 \%$ \\
\hline
\end{tabular}

Sin la opción de cambio, solo considerando la opción de abandono en cada uno de los escenarios posibles sería más atractivo la configuración dada por una planta flexible en los Estados Unidos y una planta dedicada en Colombia, esto generaría un valor esperado de utilidad equivalente a U\$76.198.789.

La tabla IV, muestra el valor presente de las utilidades considerando para cada una de ellas las respectivas opciones de cambio.

TABLA IV

VPN DE LAS UTILIDADES PARA CADA CONFIGURACIÓN INCLUYENDO OPCIÓN DE CAMBIO

\begin{tabular}{cccc}
\hline \hline Estados Unidos & Colombia & $\begin{array}{c}\text { Valor presente } \\
\text { de las utilidades }\end{array}$ & $\begin{array}{c}\text { Diferencia } \\
\text { porcentual con } \\
\text { respecto al } \\
\text { máximo }\end{array}$ \\
\hline Dedicada & Dedicada & 71.436 .365 & $10,27 \%$ \\
Flexible & Dedicada & 76.198 .789 & $3,38 \%$ \\
Dedicada & Flexible & 71.219 .891 & $10,60 \%$ \\
Flexible & Flexible & 75.982 .316 & $3,67 \%$ \\
F/D & F/D & 78.771 .987 & $0,00 \%$ \\
\hline \hline
\end{tabular}

El tener la posibilidad de cambio, si le genera valor agregado al proyecto, dado que se obtiene un valor presente de utilidad esperado equivalente a U\$78.771.987 el cual es superior a todos los demás. En la columna diferencia porcentual se captura el valor de la flexibilidad relacionando el máximo valor obtenido en la configuración con que considera el cambio respecto de cada una de las otras configuraciones, teniendo en cuenta que si no existiese dicha opción se optaría por una configuración flexible para Estados Unidos y dedicada para Colombia, entonces el valor de la opción de cambio está dado por esa diferencia es decir es equivalente a U\$2.573.198 lo cual es una diferencia significativa.

Cuando se consideran las decisiones contingentes dentro de un modelo el resultado obtenido es equivalente como mínimo al mejor de los resultados cuando dichas decisiones no son consideradas.

La tabla $\mathrm{V}$ muestra un análisis comparativo de la utilidad de la opción de cambio, con diferentes configuraciones iniciales, observándose que para obtener el máximo valor la configuración inicial debe ser la planta de Estados Unidos dedicada y la de Colombia dedicada, en este caso se evidencia que el valor de una opción de cambio para la configuración cadenas de suministro depende de las configuraciones iniciales de arranque y de las posibles decisiones que se puedan tomar dentro del horizonte en el cual se posea la opción.

TABLA V 
VALOR PRESENTE DE LAS UTILIDADES CONSIDERANDO OPCIÓN DE CAMBIO CON DIFERENTES PUNTOS DE ARRANQUE

\begin{tabular}{ccc}
\hline \hline Estados Unidos & Colombia & \\
\hline Dedicada & Dedicada & 78.771 .987 \\
Flexible & Flexible & 78.331 .289 \\
Flexible & Dedicada & 78.363 .528 \\
Dedicada & Flexible & 78.739 .173 \\
\hline \hline
\end{tabular}

A pesar de que el valor presente neto de las utilidades apunta a una configuración con opción de cambio, es interesante saber que sucede cuando se realiza un análisis asumiendo la incertidumbre de cada una de las variables presentes en esta decisión. Por los datos iniciales se sabe la volatilidad de la demanda, las tasas de cambio y el costo promedio de cambio de cada una de las plantas, dichas variables son consideradas como generadoras de riesgo.

El análisis de sensibilidad indica que las variables que más inciden en el valor de las utilidades son la volatilidad de la demanda de Estados Unidos y de la tasa de cambio, dichas variables se deben considerar y ver si la organización puede realizar algunas medidas de intervención que permitan que estas se comporten a su favor. Para las configuraciones con ambas plantas dedicadas y Estados Unidos dedicada y Colombia flexible, la variable más crítica es decir la que más influye en el valor de las utilidades es la demanda en los Estados Unidos, por lo que se debe realizar Estados Unidos, favoreciendo los aumentos de dicha variable. Mientras que para las configuraciones de Estados Unidos flexible y Colombia dedicada o ambas flexibles tanto la demanda de ambos países son variables críticas que afectar de manera similar el valor de la utilidad total, por lo que se debe centrar esfuerzos en diseñar estrategias que contribuyan al aumento de estas demandas.

Realizando el análisis de sensibilidad a la configuración con opción de cambio se obtuvo que la opción de cambio se ve favorecida por las volatilidades en las tasas de cambio en la medida que se podría aprovechar al máximo cuando se encuentre baja y dado que se cuenta con una opción de abandono, si los ingresos es un periodo determinado no superan los costos se opta por no realizar las operaciones de distribución.

Además, más del $90 \%$ de la variación del proyecto con opción de cambio se puede explicar a partir de la volatilidad en el costo de pasar de una planta dedicada a una planta flexible, por lo que esta se considera variables claves o variables estratégicas del negocio, dado que en la medida que se puedan controlar o manejar de una manera eficiente se obtendrán los mejores resultados desde el punto de vista de la utilidad.

Además del valor presente para la toma de decisiones respecto a la configuración a escoger, se consideraron otras medidas de desempeño importantes para la organización, para cada una de las configuraciones existentes se identifican 6 variables claves, dichas variables son:

El manejo administrativo: La gestión interna y externa para diferentes configuraciones en aspectos como los tributarios, de aduanas, y departamentos dentro de la organización.

Eficiencia: Se relaciona con la utilización de la capacidad disponible de las plantas para cada una de las configuraciones posibles.
Capacidad de respuesta: Se relaciona con la posibilidad de brindar un buen servicio al cliente dado que el producto se encuentra más cerca de el por lo que se esperan menos tiempos de espera

El manejo de los inventarios: Se relaciona con las estrategias de inventario para cada una de las configuraciones.

El valor presente de cada una de las configuraciones: El cual es resultado de la simulación.

El riesgo: El cual es resultado de la simulación, y se obtiene como el inverso del coeficiente de variación.

Teniendo en cuenta estas variables se aplicó el modelo AHP. Se obtuvo el vector de prioridad para criterios y el vector de prioridad total, los cuales se ilustran en las tablas VI y VII respectivamente.

TABLA VI

VECTOR DE PRIORIDAD PARA CRITERIOS DE DECISIÓN

\begin{tabular}{cc}
\hline \hline Criterio & Prioridad \\
\hline Manejo administrativo & $5,62 \%$ \\
Eficiencia & $14,85 \%$ \\
Capacidad de respuesta y servicio & $18,27 \%$ \\
al cliente & \\
Manejo del inventario & $4,13 \%$ \\
VPN esperado & $38,58 \%$ \\
Nivel de riesgo & $18,56 \%$ \\
\hline \hline
\end{tabular}

TABLA VII

VECTOR DE PRIORIDAD TOTAL

\begin{tabular}{cc}
\hline \hline Alternativas & Prioridad \\
\hline Ambas plantas dedicadas & $13,59 \%$ \\
Ambas plantas flexibles & $17,00 \%$ \\
U flexible M dedicada & $11,41 \%$ \\
U dedicada M flexible & $11,90 \%$ \\
Con opción de Cambio & $46,10 \%$ \\
\hline \hline
\end{tabular}

En las tablas VI y VII, se corrobora que dados los criterios establecidos la mejor opción es la opción de cambio seguida de las plantas opción con ambas plantas flexibles, esto se puede explicar dado que en el vector de prioridades de la compañía el factor dominante es el valor presente, seguido por el nivel de riesgo, factores en que la opción de cambio fue predominante.

\section{CONCLUSIONES Y TRABAJOS Futuros}

A través de las opciones reales, la cadena de suministro puede optimizar los precios de los factores en el mercado o tomar decisiones que mejoren medidas de desempeño como los costos o utilidades totales, esto se realiza anticipando posibles fluctuaciones que puedan perjudicar los bienes o servicios ofrecidos y demandados, por tanto, las opciones deben ser vistas como herramientas para la estabilización de los resultados y no como una fuente adicional de ingresos para la cadena.

Teniendo en cuenta los horizontes de planeación, la configuración de cadenas de abastecimiento utilizando las opciones reales se ubican más fácilmente en el horizonte de planeación táctico y operativo, dado que las opciones se presentan a medida que transcurre la operación y es muy 
complicado identificarlas en horizontes de tiempo extremadamente largos.

Desde el punto de vista de la implementación, la valoración de las opciones reales con árboles de decisión es un poco dispendioso, dado la gran cantidad de elementos que pueden surgir por cada uno de los nodos, a razón de $2^{n}$ donde $\mathrm{n}$ es el número de activos subyacentes, en un problema real la implementación de árboles binomiales es compleja y se deben buscar otras herramientas de solución, como la valoración por Black-Scholes, aunque esto puede derivar en modelos matemáticos complejos que en muchos casos son difíciles de entender de una manera intuitiva.

El método de opciones reales no reemplaza el método de valoración por flujo de caja, antes bien complemente este teniendo en cuenta las posibilidades de alterar el rumbo en el futuro dentro de la configuración de una cadena de suministro.

Debido a que las estructuras que se generan para los diferentes tipos de opciones reales presentes en una cadena de suministro generalmente no son lineales, para su valoración es más sencillo, la utilización de modelos de simulación, antes que los modelos de optimización.

El valor de una opción de cambio en cadenas de suministro depende entre otras cosas del valor de cambio de un tipo de configuración a otra, y de la configuración de arranque, por lo que se deben desarrollar algoritmos que permitas evaluar todas las posibles combinaciones de arranque.

El AHP en cadenas de suministros permite modelar la interacción simultánea de diversos factores en situaciones complejas y no estructuradas tal como la configuración de las cadenas de suministros, su potencialidad radica en que se pueden evaluar criterios cuantitativos y cualitativitos y generar una medida de desempeño consistente para cada una de las posibles configuraciones, con lo que se puede tomar una decisión que cumpla con los objetivos estratégicos de la organización [22].

Si la cantidad de configuraciones posibles en la cadena de suministro es considerablemente grande, se evidencia una complejidad práctica a la hora de implementar la metodología AHP, dado lo exhaustivo que sería el proceso de calificación, en este sentido se debería realizar un proceso de depuración, con el objetivo de reducir el número de configuraciones existentes, o por el contrario desarrollar metodología que hagan este proceso más eficiente, un primer acercamiento es la programación por metas, aunque el proceso es dificultoso debido a las gran cantidad de calificaciones que se deben realizar.

Uno de los supuestos fundamentales del modelo desarrollado es que las variables de riesgo del modelo se comportaban de manera independiente, un estudio siguiente, debe incluir el efecto de las correlaciones de dichas variables en el valor de las opciones de configuración.

Se han considerado opciones de manera independiente, sin tener en cuenta las interrelaciones entre ellas, por lo que un trabajo futuro sugiere la valoración de las opciones dentro de las cadenas de suministro, como un portafolio de opciones y no como opciones independientes.

Una de las herramientas utilizadas por organizaciones que tienen operaciones en diversos países son los precios de transferencia entre los eslabones de su cadena, por lo que un estudio posterior debe tener en cuenta el efecto de dichos precios en el valor de las opciones reales presentes la red de suministro.

Es importante destacar que el valor de una opción depende en gran medida del cálculo de la volatilidad, y aunque en el presente trabajo se utilizó la prueba Samuelson citada por [23], que estima la volatilidad como la variación de los rendimientos logarítmicos de los valores presentes del proyecto, un trabajo posterior evaluar el efecto que tiene el utilizar otras pruebas en dicho cálculo, ya que diversos autores indican que dicha prueba subestima la volatilidad y por lo tanto incide en el valor de la opción.

Un trabajo futuro debe considerar el efecto del costo de la capacidad ociosa cuando se decide utilizar la opción de abandono en la utilidad global de la organización.

Sería interesante comparar los resultados obtenidos con los que se pudieran obtener a través de un modelo que pueda ser resuelto mediante optimización estocástica.

\section{REFERENCIAS}

[1] R. Pellegrino, N. Costantino, and D. Tauro, "Supply Chain Finance: A supply chain-oriented perspective to mitigate commodity risk and pricing volatility," J. Purch. Supply Manag., vol. 25, no. 2, pp. 118133, 2019. DOI: https://doi.org/10.1016/j.pursup.2018.03.004

[2] S. Chopra, P. Meindl, and D. V. Kalra, Supply chain management: strategy, planning, and operation, vol. 232. Pearson Boston, MA, 2013.

[3] W. Klibi, A. Martel, and A. Guitouni, "The design of robust valuecreating supply chain networks: A critical review," Eur. J. Oper. Res., vol. 203, no. 2, pp. 283-293, Jun. 2010. DOI: https://doi.org/10.1016/j.ejor.2009.06.011

[4] R. Rajesh, "A grey-layered ANP based decision support model for analyzing strategies of resilience in electronic supply chains," Eng. Appl. Artif. Intell., vol. 87, p. 103338, 2020. DOI: https://doi.org/10.1016/j.engappai.2019.103338

[5] R. Narasimhan and S. Talluri, "Perspectives on risk management in supply chains," J. Oper. Manag., vol. 27, no. 2, pp. 114-118, Apr. 2009. DOI: https://doi.org/10.1016/j.jom.2009.02.001

[6] M. Pournader, A. Kach, and S. Talluri, "A Review of the Existing and Emerging Topics in the Supply Chain Risk Management Literature," Decis. Sci., vol. 51, no. 4, pp. 867-919, 2020. DOI: https://doi.org/10.1111/deci.12470.

[7] D. Neiger, K. Rotaru, and L. Churilov, "Supply chain risk identification with value-focused process engineering," J. Oper. Manag., vol. 27, no. 2, pp. 154-168, 2009. DOI: https://doi.org/10.1016/j.jom.2007.11.003

[8] M. Louis and M. Pagell, "Categorizing supply chain risks: review, integrated typology and future research," in Revisiting supply chain risk, Springer, 2019, pp. 329-366. DOI: https://doi.org/10.1007/9783-030-03813-7_20

[9] K. Ganguly and G. Kumar, "Supply chain risk assessment: a fuzzy AHP approach," Oper. Supply Chain Manag. An Int. J., vol. 12, no. 1, pp. 1-13, 2019. . DOI: http://doi.org/10.31387/oscm0360217

[10] P. Trkman and K. McCormack, "Supply chain risk in turbulent environments-A conceptual model for managing supply chain network risk," Int. J. Prod. Econ., vol. 119, no. 2, pp. 247-258, 2009. DOI: https://doi.org/10.1016/j.ijpe.2009.03.002

[11] R. Oger, M. Lauras, B. Montreuil, and F. Benaben, "A decision support system for strategic supply chain capacity planning under uncertainty: conceptual framework and experiment," Enterp. Inf. Syst., pp. 1-45, 2020.DOI: https://doi.org/10.1080/17517575.2020.1793390

[12] R. Sreedevi and H. Saranga, "Uncertainty and supply chain risk: The moderating role of supply chain flexibility in risk mitigation," Int. J. 
Prod. Econ., vol. 193, pp. 332-342, 2017.DOI: https://doi.org/10.1016/j.ijpe.2017.07.024

[13] H. Nembhard, L. Shi, and M. Aktan, "A real-options-based analysis for supply chain decisions," IIE Trans., vol. 37, no. February 2015, pp. 945-956, 2005.DOI: DOI: 10.1080/07408170591008073

[14] H. K. Chan and F. T. S. Chan, "A review of coordination studies in the context of supply chain dynamics," Int. J. Prod. Res., vol. 48, no. $\begin{array}{llll}10, & \text { pp. } & 2793-2819, & \text { 2010.DOI: }\end{array}$ https://doi.org/10.1080/00207540902791843

[15] M. A. Brach, Real options in practice, vol. 198. John Wiley \& Sons, 2003.

[16] R. S. Vila, "Real options analysis in real estate investments and developments." Universitat Autònoma de Barcelona, 2019.

[17] H. T. J. Smit and L. Trigeorgis, Strategic investment: Real options and games. Princeton University Press, 2012.

[18] D. F. M. Duque and P. C. Manyoma, "La evaluación de proyectos de inversión mediante opciones reales: aspectos conceptuales," Ing. y Compet., vol. 3, no. 1, pp. 7-18, 2001.DOI: 10.25100/iyc.v3i1.2332

[19] H. L. Lee, "Aligning supply chain strategies with product uncertainties," Calif. Manage. Rev., vol. 44, no. 3, pp. 105-119, 2002.

[20] J. Seppälä, L. Basson, and G. A. Norris, "Decision analysis frameworks for life-cycle impact assessment," J. Ind. Ecol., vol. 5, no. 4 , pp. 45-68, 2001.

[21] T. L. Saaty, "Decision making with the analytic hierarchy process," Int. J. Serv. Sci., vol. 1, no. 1, pp. 83-98, 2008.

[22] T. L. Saaty, "The analytic hierarchy and analytic network processes for the measurement of intangible criteria and for decision-making," in Multiple criteria decision analysis, Springer, 2016, pp. 363419.DOI: https://doi.org/10.1007/978-1-4614-7279-7

[23] T. Copeland and V. Antikarov, "Real options: A practitioner's guide, revised edition," Texere, ISBN-10, vol. 1587991861, 2003.

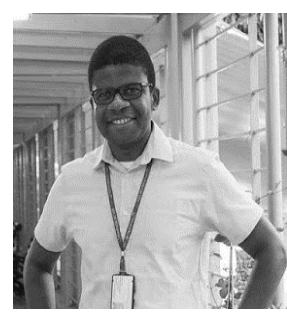

Victor Javier Jiménez Carabalí. Profesor Asistente. Departamento de Contabilidad y Finanzas, Universidad del Valle. Doctorado en Engenharia Industrial e Sistemas, Universidade do Minho. Magister en Ingeniería e Ingeniero Industrial de la Universidad del Valle. Áreas de Interés: Gestión Estratégica de Costos, Ingeniería Económica. Evaluación Financiera de Proyectos. Gestión Financiera de Entidades del Sector Salud.

ORCID: https://orcid.org/0000-0001-9377-5670

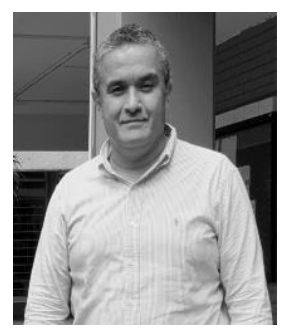

Diego Fernando Manotas Duque. Profesor Titular. Escuela de Ingeniería Industrial, Universidad del Valle. Doctor en Ingeniería, Universidad del Valle. Magíster en Gestión de Empresas, Universidad de Chile. Ingeniero Industrial y Especialista en Finanzas, Universidad del Valle. Áreas de interés: Finanzas, Ingeniería Económica. Análisis y Gestión de Riesgo Financiero. Evaluación Financiera de Proyectos. Gestión Financiera de Entidades de Economía Solidaria. Ingeniería Financiera.

ORCID: https://orcid.org/0000-0003-0148-9840

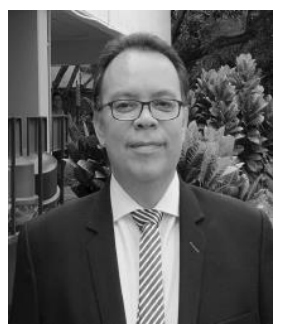

Leonardo Rivera Cadavid. Profesor Asistente. Escuela de Ingeniería Industrial, Universidad del Valle. Ph.D. in Industrial and Systems Engineering, Virginia Polytechnic Institute and State University. M.Sc. Industrial and Systems Engineering, Georgia Institute of Technology. Ingeniero Industrial Universidad del Valle. Áreas de interés: Logística. Diseño de Centros de Distribución. Investigación de Operaciones Aplicada. Lean Manufacturing. Modelación de Cadenas de Abastecimiento. Programación y Algoritmos en Ingeniería Industrial.

ORCID: https://orcid.org/0000-0001-9942-5188 